\title{
HYPERGLYCAEMIA AND OUTCOME IN CRITICALLY ILL CHILDREN WITH CENTRAL NERVOUS SYSTEM ILLNESS
}

\author{
Manikfan Abdul Azeez ${ }^{1}$, Machinary Puthenpurayil Jayakrishnan²
}

${ }^{1}$ Consultant Paediatrician, Department of Paediatrics, BM Hospital, Pulikkal, Malappuram, Kerala, India. ${ }^{2}$ Additional Professor, Department of Paediatrics, Government Medical College, Kozhikode, Kerala, India.

\author{
BACKGROUND \\ ABSTRACT \\ Hyperglycaemia is common in children admitted in the intensive care unit and this has been reported to be associated with \\ increased mortality and morbidity. \\ Aims- To study the correlation between hyperglycaemia and outcome in critically ill children with central nervous system disease \\ admitted in the Paediatric Intensive Care Unit (PICU) of a tertiary hospital in South India.
}

\section{MATERIALS AND METHODS}

A prospective observational study was conducted from August 2011 to January 2012 in the PICU of Government Medical College, Kozhikode, a major referral hospital in North Kerala. The Institutional Ethics Committee approved the study. Children in the age group of 29 days to 12 years with CNS disease admitted to the PICU were included. Blood glucose was measured with glucometer at admission, at 6 hours and 12 hourly for first 24 hours or till the child attained normoglycaemia. The children were assessed for outcome till discharge from the hospital. Outcome was assessed on the basis of Glasgow Coma Scale (GCS), neurological deficit or death.

\section{RESULTS}

Out of 226 children, 33 children (14.6\%) had hyperglycaemia at hospitalisation. Two children died. No significant association was found between blood sugar at admission and GCS or neurological status at discharge.

\section{CONCLUSION}

There is a statistically significant correlation between duration of hyperglycaemia and complete recovery without any neurological deficit in children with neurocritical illness. No significant association was found between presence, severity or duration of hyperglycaemia and outcome in children with neurocritical illness.

\section{KEY WORDS}

Hyperglycaemia, Outcome, Critical Illness, CNS.

HOW TO CITE THIS ARTICLE: Azeez MA, Jayakrishnan MP. Hyperglycaemia and outcome in critically ill children with central nervous system illness. J. Evolution Med. Dent. Sci. 2018;7(28):3148-3150, DOI: 10.14260/jemds/2018/708

\section{BACKGROUND}

As a part of physiological response to stress, it is common to find hyperglycaemia in children admitted in the intensive care unit. It has been observed that high blood sugar in the critically ill child is associated with prolonged ICU stay and mortality. ${ }^{1}$ This includes infections and neurological disorders, sepsis and traumatic brain injury. On the other hand, tight glucose control in the ICU has been proposed to be associated with adverse outcomes including increased ICU stay and mortality. Studies related to correlation between hyperglycaemia and outcome in children with critical illness due to Central Nervous System (CNS) infection are scarce in the literature. The present study was conducted to look into this correlation in children admitted to the Paediatric Intensive Care Unit (PICU) of a tertiary care hospital in South India.

'Financial or Other Competing Interest': None.

Submission 31-05-2018, Peer Review 24-06-2018,

Acceptance 30-06-2018, Published 09-07-2018.

Corresponding Author:

Machinary Puthenpurayil Jayakrishnan,

Additional Professor,

Department of Paediatrics,

Government Medical College, Kozhikode, Kerala, India.

E-mail: mp.jayakrishnan@gmail.com

DOI: $10.14260 /$ jemds $/ 2018 / 708$

\section{(c) $(1) \ominus$}

\section{MATERIALS AND METHODS}

This prospective observational study was conducted in the PICU of Government Medical College, Kozhikode for a period of 6 months between August 2011 and January 2012.

All children admitted in the PICU with CNS disease in the age group of 29 days to 12 years were included in the study. Children with pre-existing diabetes were excluded. Informed consent was obtained from parents.

'Critically ill' children were defined as those children with CNS diseases requiring ICU admission such as status epilepticus; intracranial space occupying lesion (ICSOL); CNS infections like meningitis, encephalitis, meningoencephalitis or brain abscess. Detailed clinical examination was conducted.

Blood glucose was measured with glucometer at admission, at 6 hours and $12^{\text {th }}$ hourly for first $24 \mathrm{hrs}$. or till the child attained normoglycaemia. Hyperglycaemia was defined as blood glucose $>140 \mathrm{mg} / \mathrm{dL}$. Hyperglycaemia was categorised into 4 groups- Mild $(140-200 \mathrm{mg} / \mathrm{dL})$, moderate $(200-250 \mathrm{mg} / \mathrm{dL})$, severe $(250-300 \mathrm{mg} / \mathrm{dL})$ and profound (> $300 \mathrm{mg} / \mathrm{dL}$ ). Total and differential white cell count, platelet count, serum electrolytes, creatinine, urea, liver function tests, blood and urine, and cerebrospinal fluid (CSF) examination and cultures were done at admission. The children were assessed for outcome till discharge from the hospital. None of the children were treated with insulin. 
Outcome was assessed on the basis of Glasgow Coma Scale (GCS) status and neurological deficit. Outcome was categorised as complete recovery without any neurological deficit, recovery with neurological deficit or death.

\section{RESULTS}

Out of 226 cases of CNS diseases admitted in PICU, 33 children had hyperglycaemia at hospitalisation and were included in the study. The results are summarised in Table 1.

Majority of patients with hyperglycaemia were infants. Children in the $1-5$ year and $5-12$ years of age group constituted. None of the children had hypoglycaemia. Male female ratio was 2.1: 1 with 17 males and 16 females.

Seizure was the commonest presentation, comprising of 30 children. Other symptoms included altered sensorium, headache or poor feeding.

Majority of children had mild hyperglycaemia. Moderate hyperglycaemia was present in 4 children, severe and profound hyperglycaemia in 3 each.

In some children blood glucose normalised within $12 \mathrm{hrs}$., for some children within 12 - 24 hrs., other children within $24-48$ hrs. $3 \%>48$ hrs. and $3 \%$ had persistent hyperglycaemia. The boy with persistent hyperglycaemia died on the 4th day of hospitalisation.

Majority of children had a GCS score of $>8$ at admission. GCS score of all children who survived was complete (15/15) at the time of discharge from the hospital, irrespective of the initial blood sugar status.

Out of the 33 children included in the study, 2 died. The first was a 12-year-old boy with arterial thrombosis and right hemiparesis. He had mild hyperglycaemia at admission, which persisted till his death on the 4th day of hospitalisation. The other child was a 36-day-old male baby with meningitis and status epilepticus. His blood sugar had normalised within 24 hours, but expired on the 12th day of hospitalisation.

\begin{tabular}{|c|c|}
\hline & No (\%) \\
\hline \multicolumn{2}{|l|}{ Age } \\
\hline$\leq 1$ year & $14(42.4)$ \\
\hline $1-5$ years & $11(33.3)$ \\
\hline $6-12$ years & $8(24.2)$ \\
\hline \multicolumn{2}{|l|}{ Gender } \\
\hline Male & $17(51.5)$ \\
\hline Female & $16(48.5)$ \\
\hline \multicolumn{2}{|l|}{ Clinical presentation } \\
\hline Seizure & $22(66.7)$ \\
\hline Seizure with fever & $8(24.2)$ \\
\hline Weakness & $1(3)$ \\
\hline Others & $2(6.1)$ \\
\hline \multicolumn{2}{|l|}{ GCS at Admission } \\
\hline$\leq 7$ & $8(24.2)$ \\
\hline$\geq 8$ & $25(75.8)$ \\
\hline \multicolumn{2}{|c|}{ Blood Sugar at Admission } \\
\hline $140-200$ & $23(69.7)$ \\
\hline $201-250$ & $4(12.1)$ \\
\hline $251-300$ & $3(9.1)$ \\
\hline$>300$ & $3(9.1)$ \\
\hline \multicolumn{2}{|c|}{ Neurologic Deficit at Admission } \\
\hline Present & $11(33.3)$ \\
\hline Absent & $22(66.7)$ \\
\hline \multicolumn{2}{|c|}{ Time taken to Normalise Blood Sugar } \\
\hline$<12$ hours & $14(42.4)$ \\
\hline $12-24$ hours & $10(30.3)$ \\
\hline
\end{tabular}

\begin{tabular}{|c|c|}
\hline $24-48$ hours & $7(21.2)$ \\
\hline $48-72$ hours & $1(3)$ \\
\hline Persistently elevated & $1(3)$ \\
\hline GCS at Discharge & $1(3)$ \\
\hline 9 & $1(3)$ \\
\hline 12 & $1(3)$ \\
\hline 14 & $28(84.8)$ \\
\hline 15 & $2(6.1)$ \\
\hline Neurologic Deficit at Discharge & $29(87.9)$ \\
\hline Present & \\
\hline Absent & $29(87.9)$ \\
\hline Outcome & $2(6.1)$ \\
\hline Complete recovery & $2(6.1)$ \\
\hline Recovery with neurologic deficit \\
\hline Death \\
\hline \multicolumn{2}{|c|}{ Clinical Characteristics (Total No. 33) } \\
\hline
\end{tabular}

\section{DISCUSSION}

Our study included critically ill children with CNS disease admitted in the PICU of a large referral centre in South India. We specifically looked for association of hyperglycaemia on outcome in children with neurocritical illness. There have been very few published studies on critical illness hyperglycaemia in neurocritical illness. ${ }^{2,3}$

The incidence of hyperglycaemia in the present study was $14.6 \%$. In a prospective study in adults with bacterial meningitis, the incidence of hyperglycaemia was reported to be $69 \%$. The reported incidence of hyperglycaemia in critically ill children ranges from $17 \%$ to $86 \%$, depending on blood glucose cut-off, number of measurements, time of blood glucose evaluation and patient population studied. 4,5

In our study outcome measures selected included complete recovery without neurological deficit, GCS status, neurological deficits or death.

There was no significant association between blood glucose level at the time of admission and occurrence of neurological deficit, death or low GCS status in this study.

Likewise, there was no significant association between duration of hyperglycaemia and occurrence of neurological deficit, death or low GCS status. The possible reason for this could be low mortality (6\%) and low occurrence of neurological deficit at discharge (6.4\%) in our cohort.

We could not establish any correlation between severity of hyperglycaemia at hospitalisation and duration of hyperglycaemia, as most cases with severe hyperglycaemia $(250-300 \mathrm{mg} / \mathrm{dL}$ or more) attained normoglycaemic range in a short period of time ( $<24$ hours). Regarding GCS status irrespective of GCS status or blood glucose at admission or duration of hyperglycaemia, all children who survived had full GCS at the time of discharge. This is almost same in case of neurological deficit. Most children who had neurological deficit at admission were normal at the time of discharge (93.5\% of those survived). Only 2 children had any neurological deficit at discharge. Both these children had mild hyperglycaemia (<200 mg/dL) and duration of hyperglycaemia was $<24$ hours. Both these children were having tuberculous meningitis. Thus, we could not establish any significant association between hyperglycaemia and occurrence of neurological deficit. Most of the children (94\%) in this study had their blood glucose level normalised within 48 hours of hospitalisation. 
We found a statistically significant correlation between lesser duration of hyperglycaemia and chance of complete recovery without neurological deficits, even though the total number of children with hyperglycaemia after 48 hours was only $6 \%$.

Hyperglycaemia in critical illness has been found to be associated with greater in-hospital mortality and longer PICU stay in many studies. ${ }^{1,4}$ Duration of hyperglycaemia has also been reported to be independently associated with mortality in critically ill children. ${ }^{4}$

On the other hand, tight glycaemic control using insulin has been found to be associated with no mortality benefit and an increased risk of hypoglycaemia. ${ }^{6,7,8}$ None of the children were treated with insulin in our cohort.

Blood glucose variability, rather than hyperglycaemia alone was found to be associated with multiorgan dysfunction and increased ICU stay in some studies.9,10,11 Blood glucose variability as a factor related to adverse outcome was not looked for in our study, as most of the children (94\%) had their glucose level normalised within 48 hours of admission.

In this study all the children who survived had full GCS at the time of discharge, irrespective of severity of hyperglycaemia at admission or duration of hyperglycaemia or disease status.

\section{CONCLUSION}

There is a statistically significant correlation between duration of hyperglycaemia and complete recovery without any neurological deficit in children with neurocritical illness. Lesser duration of hyperglycaemia was associated with more chance of complete recovery. We could not find any significant association between presence, severity or duration of hyperglycaemia and outcome in children with neurocritical illness. The major limitation of the study is the small sample size and having no control group.

\section{REFERENCES}

[1] Bhutia TD, Lodha R, Kabra SK. Abnormalities in glucose homeostasis in critically ill children. Pediatr Crit Care Med 2013;14(1):e16-25.

[2] Schut ES, Westendorp WF, de Gans J, et al. Hyperglycemia in bacterial meningitis: a prospective cohort study. BMC Infect Dis 2009;9:57.

[3] Green DM, O'Phelan KH, Bassin SL, et al. Intensive versus conventional insulin therapy in critically ill neurologic patients. Neurocrit Care 2010;13(3):299306.

[4] Srinivasan V, Spinella PC, Drott HR, et al. Association of timing, duration \& intensity of hyperglycemia with ICU mortality in critically ill children. Pediatr Crit Care Med 2004;5(4):329-36.

[5] Faustino EV, Apkon M. Persistent hyperglycemia in critically ill children. J Pediatr 2005;146(1):30-4.

[6] Macrae D, Grieve R, Allen E, et al. A randomized trial of hyperglycemic control in pediatric intensive care. $\mathrm{N}$ Engl J Med 2014;370(2):107-18.

[7] Chen L, Li T, Fang F, et al. Tight glycemic control in critically ill pediatric patients: a systematic review and meta-analysis. Crit Care 2018;22(1):57.

[8] Kandil SB, Spear D, Thomas NJ, et al. Retrospective outcomes of glucose control in critically ill children. J Diabetes Sci Technol 2013;7(5):1220-8.

[9] Hirshberg E, Larsen G, Van Duker H. Alterations in glucose homeostasis in the pediatric intensive care unit: Hyperglycemia and glucose variability are associated with increased mortality and morbidity. Pediatr Crit Care Med 2008;9(4):361-6.

[10] Naranje KM, Poddar B, Bhriguvanshi A, et al. Blood glucose variability and outcomes in critically ill children. Indian J Crit Care Med 2017;21(3):122-6.

[11] Rake AJ, Srinivasan V, Nadkarni V, et al. Glucose variability and survival in critically ill children: allostasis or harm? Pediatr Crit Care Med 2010;11(6):707-12. 